This item was submitted to Loughborough's Research Repository by the author.

Items in Figshare are protected by copyright, with all rights reserved, unless otherwise indicated.

\title{
Modelling radiation damage at grain boundaries in fcc nickel and Ni-based alloy using long time scale dynamics techniques
}

PLEASE CITE THE PUBLISHED VERSION

http://dx.doi.org/10.1016/j.nimb.2012.10.027

PUBLISHER

(C) Elsevier

VERSION

AM (Accepted Manuscript)

LICENCE

CC BY-NC-ND 4.0

\section{REPOSITORY RECORD}

Tooq, Zainab Al, and Steven D. Kenny. 2019. "Modelling Radiation Damage at Grain Boundaries in Fcc Nickel and Ni-based Alloy Using Long Time Scale Dynamics Techniques”. figshare.

https://hdl.handle.net/2134/11623. 
This item was submitted to Loughborough's Institutional Repository (https://dspace.lboro.ac.uk/) by the author and is made available under the following Creative Commons Licence conditions.

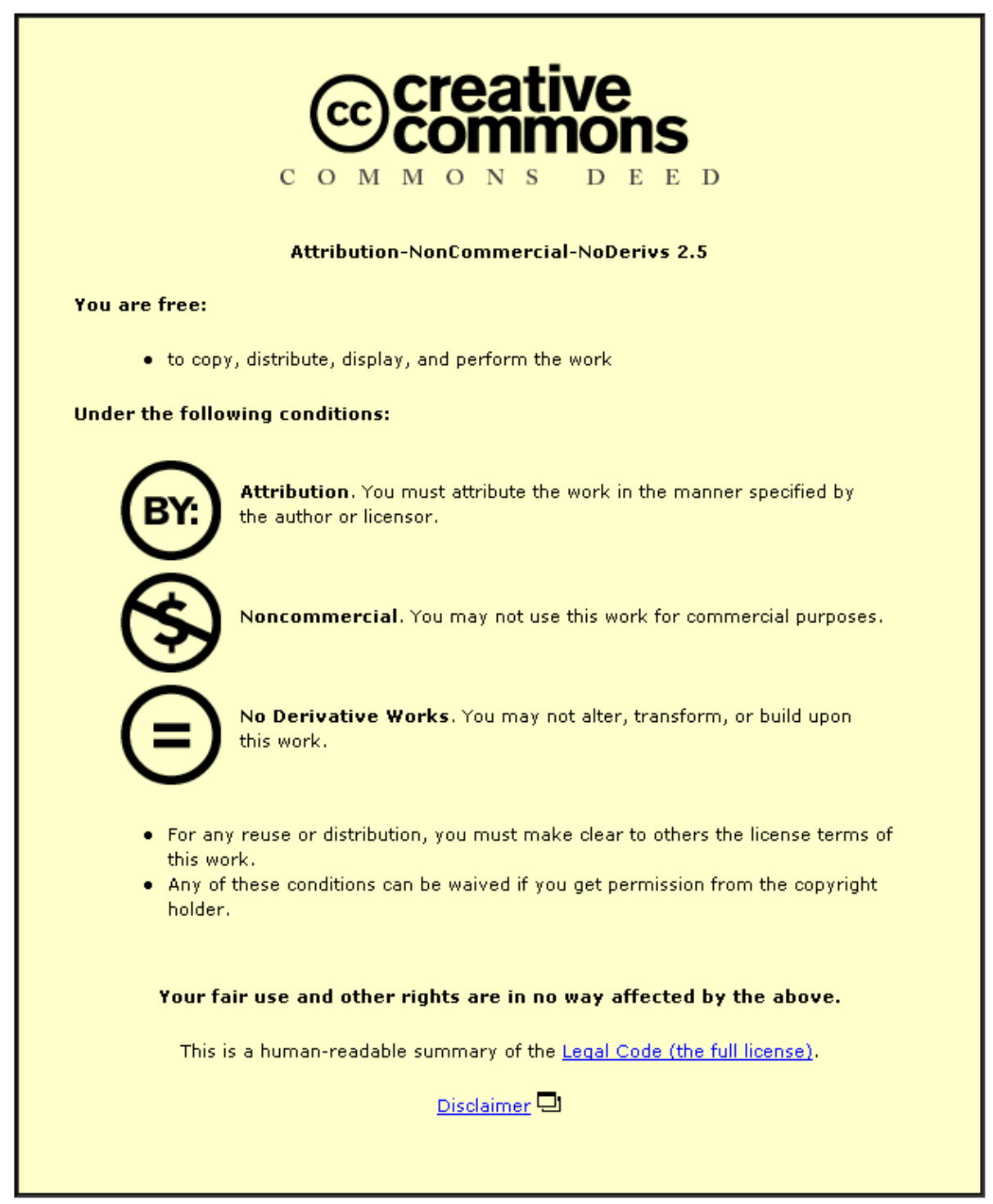

For the full text of this licence, please go to: http://creativecommons.org/licenses/by-nc-nd/2.5/ 


\title{
Modelling radiation damage at grain boundaries in fcc Nickel and Ni-based alloy using Long Time Scale Dynamics Techniques
}

\author{
Zainab Al Tooq, Steven D Kenny \\ Department of Mathematical Sciences, Loughborough University, Leicestershire, LE11 3TU, UK
}

\begin{abstract}
The long timescale evolution of radiation damage near grain boundaries (GB) in fcc Ni and a Ni-Cr alloy has been investigated. Molecular Dynamics (MD) combined with the on-the-fly Kinetic Monte Carlo (otf-KMC) has been used to study the evolution of defects in bulk, $\Sigma 3$ and $\Sigma 5$ GB systems resulting from $1 \mathrm{keV}$ collision cascades. Results show that both interstitials and vacancies are attracted to GBs, that vacancy and interstitial clustering is observed in the bulk and vacancy clustering in the GB systems.
\end{abstract}

\section{Introduction}

Due to its ability to change the physical properties of materials there have been numerous studies of the effect of radiation damage on materials. One of the important effects of radiation on materials is radiation induced segregation (RIS), especially near grain boundaries (GB), as it can lead to effects such as the embrittlement of the material. Ni-based alloys represent an interesting class of materials for next generation nuclear power plants. This is due the presence of $\gamma \prime$ phase in Ni-based alloys which gives them high temperature strength and creep and oxidation resistance.

In recent years computer simulation has become an important tool to investigate such problems. MD, for instance, is an extremely powerful tool to address many problems related to radiation damage and has given insight into numerous aspects regarding radiation damage $[1,2]$. The limitation of the simulation time attainable, however, means that the evolution of radiation damage cannot be simulated over realistic timescales, which drives the need for other techniques. On-the-fly kinetic Monte Carlo (otf-KMC) [3, 4, 5, 6] on the other hand allows modelling of complex systems, as it calculates the barriers at each step, and allows much longer timescales to be modelled, as it only follows diffusion events. Therefore using MD to model the initial phase of a collision cascade followed by otf-KMC to model the subsequent diffusion of defects is an extremely powerful tool for modelling the long time evolution of cascades.

Overall there is a large body of work aimed understanding the effect of radiation on Ni-based materials especially at grain boundaries [7]. Microstructure evolution in irradiated austenitic Fe-Cr-Ni has also been studied [8]. The study of RIS in this work showed enrichment of $\mathrm{Ni}$ and depletion of $\mathrm{Cr}$ at grain boundaries.

In this work we study the diffusion of vacancies near GBs and the evolution of defects after $1 \mathrm{keV}$ collision cascades in

Email address: Z.Al-Tooq@lboro.ac.uk (Zainab Al Tooq) bulk, $\Sigma 3$ and $\Sigma 5$ GB systems for the fcc $\mathrm{Ni}$ and a Ni-Cr binary alloy.

\section{Method}

Fcc Ni and a Ni-Cr binary alloy (80 at\% Ni 20 at\% Cr) have been studied in this work. The bulk, symmetrical tilt $\Sigma 5$ and $\Sigma 3$ grain boundaries have been studied for both materials. Systems modelled contained approximately $55 \mathrm{k}$ atoms with periodic boundary conditions in 3 dimensions for the bulk system and in the plane of GB with the top few layers fixed on the edges parallel to the GB for the GB systems. The GB systems were fully relaxed including allowing translations in the $x, y$ and $z$ directions to minimize the grain boundary energy. For the GBs in $\mathrm{Ni}-\mathrm{Cr}$, the minimum-energy $\mathrm{GB}$ in pure $\mathrm{Ni}$ is found first and then the required percentage of $\mathrm{Cr}$ is distributed randomly in the system. The system is relaxed after that holding GB separations fixed.

The Mishin 2004 [9] potential has been used to describe NiNi interactions, The Olsson 2005 [10] potential for $\mathrm{Cr}-\mathrm{Cr}$ interactions and Giovanni 2010 [11] potential has been used to represent $\mathrm{Ni}-\mathrm{Cr}$ interactions. The three potentials have been splined to the ZBL potential [12] to correctly describe small atomic separations. The cutoff distances used to connect the pair potential to the spline function and the spline function to the ZBL potential are: $0.6 \AA$ and $1.2 \AA$ for $\mathrm{Ni}-\mathrm{Ni}, 0.5 \AA$ and 1.2 $\AA$ for $\mathrm{Cr}-\mathrm{Cr}, 0.8 \AA$ and $1.95 \AA$ for $\mathrm{Ni}-\mathrm{Cr}$ potential.

The modelling carried out can be divided to two main stages. The first stage was modelling collision cascades to the system and the second stage was modelling the evolution of the defects that resulted from the first stage.

Collision cascades are initialized by imparting kinetic energy to an atom, called the primary knock-on atom (PKA), in a certain direction. An energy of $1 \mathrm{keV}$ was used to perform the collision cascades. The PKA has been chosen randomly and in case of the GB the direction of the collision cascades has been directed towards the GB. The initial collision cascade phase is 
modelled using MD, with a velocity Verlet [13] algorithm used to evolve the system. The collisions runs for $20 \mathrm{ps}$ with variable time step depending on the kinetic energy and potential energy within the system. All collisions cascades in this work were carried out at $0 \mathrm{~K}$ so no thermostat has been applied during the MD simulations.

Otf-KMC has been used to model the evolution of defects after collision cascades. The otf-KMC algorithm starts by identifying defects, which is done by comparing the system after a collision cascade to the reference system. Once these are identified, large numbers of searches are initialized to find the unique saddles points using the Activation and Relaxation Technique $[5,6]$. After finding the approximate saddles, the climbing image nudged elastic band method (CI-NEB) [14] was used to calculate the barriers for each transition found. The rate of each transition is calculated using Arrhenius equation (1):

$$
\text { Escape Frequency }=v \exp \left(-E_{b} / k_{b} T\right)
$$

where $v$ is the transition prefactor and is taken to be $10^{13} \mathrm{~s}^{-1}$, $k_{B}$ is Boltzmann constant ant $T$ is the temperature. The temperature for the evolution of the system was taken to be $500 \mathrm{~K}$ as this is representative of what would be found in a next generation reactor.

A random number is generated between 0 and the sum of the rates for all transitions and a transition is chosen by stepping all transitions cumulatively until the random number is exceeded. The time is accumulated by (2):

$$
\text { Time }=-\frac{\log _{e}(x)}{\sum_{i=1}^{i=N} \text { Rate }_{i}}
$$

where $x$ is a random number between 0 and 1 and $N$ is the total number of transitions found at the step. Finally the system is evolved by the chosen transition and all the processes repeated again.

\section{Results and discussion}

We have investigated the migration of the vacancy to the GB, this has been achieved by creating a vacancy and modelling its migration using otf-KMC. The vacancy migrates to the GB in almost all cases with a typical diffusion barrier for the vacancy of $0.9 \mathrm{eV}$. For instance, a vacancy created at distance of $20 \AA$ from the GB took $0.846 \mathrm{~ms}$ to reach the GB and $2.23 \mu$ s from distance of $5 \AA$. In the cases where the vacancy doesn't diffuse to the GB, it diffused to the fixed boundary conditions and got trapped.

MD along with otf-KMC has been used to model the evolution of defects resulting from $1 \mathrm{keV}$ cascades in bulk, $\Sigma 3$ and $\Sigma 5$ GB systems for both fcc Ni and the Ni-Cr binary alloy. For the bulk system, a vacancy cluster formed in the system during the MD phase. During the otf-KMC, there was little change in the number or location of the defects. This was due to the movement within the vacancy cluster with very low barriers, which

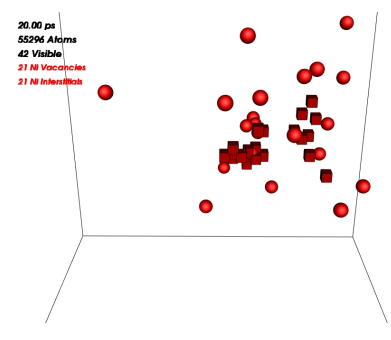

(a)

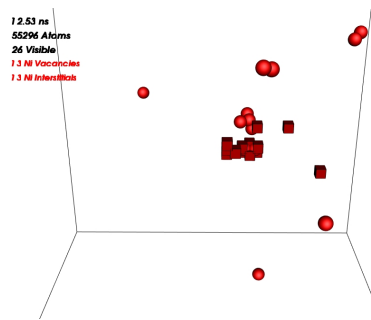

(b)
Figure 1: Evolution of radiation damage in the fcc Ni bulk system, (a) shows system after MD simulation, (b) after otf-KMC (In all figures sphere is an interstitial and cube is a vacancy).

prevented other transitions happening in the system. We studied the transitions happened over that time and we found that these movements within the cluster had barriers of less than 0.2 $\mathrm{eV}$ and atoms moved less than $1.5 \AA$. Furthermore, we studied the other transitions that could have happened to make sure that none of the other transitions satisfied these criteria. We blocked these transitions after which we could see that interstitials are more mobile than vacancies and lots of recombination between vacancies and interstitials happened. Figure (1) shows the system after the collision cascade containing 42 defects and the system after the otf-KMC with only 26 defects after $12.53 \mathrm{~ns}$.

For the $\Sigma 3$ system the twin boundary, as expected, did not have a big influence on how defects were distributed in the system or their migration. The defects were scattered in the system after the collision cascade. During the otf-KMC simulation a number of vacancy-interstitial recombinations happened in the system, before a vacancy cluster formed which resulted in the simulation being dominated by vacancy hops in the cluster. After we blocked these very low barriers, with the previous method explained for the bulk system, more recombinations occurred to leave a system of only 8 defects after $15.2 \mu \mathrm{s}$, originally there were 36 defects after the collision cascade.

The GB in the $\Sigma 5$ system has more influence on the distribution of defects in the system. Defects accumulated at the GB during the collision cascades, as shown in figure (2(a)). During the otf-KMC the mobility of interstitials was observed to be much higher than that of the vacancies. A number of defects annihilated due to recombinations between vacancies and interstitials. The vacancies were found to be attracted to the GB if they were near the GB. After an otf-KMC time of $4.93 \mu$ s 16 recombinations had occurred between vacancies and interstitials as shown in figure(2(b)).

For the bulk alloy system the observed transitions had higher barriers than those for the pure Ni system. A vacancy cluster resulted from the collision cascades as was seen for the pure fcc Ni case. The cluster and a few dumbbells are still present after running for $11 \mathrm{~ms}$ in the otf-KMC, with the results similar to the pure $\mathrm{Ni}$ case.

The twin boundary in the $\Sigma 3$ alloy system was found to have more influence on defects than for the pure Ni system. Figure (3) shows a snapshot of the system immediately after the collision cascades and after the otf-KMC. Very little recombination is seen to occur in the $242 \mu$ s simulated and their is a far greater 
accumulation of defects at the GB.

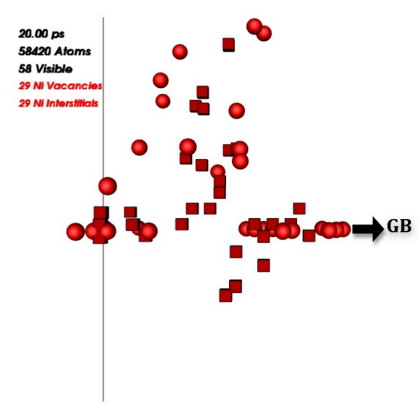

(a)

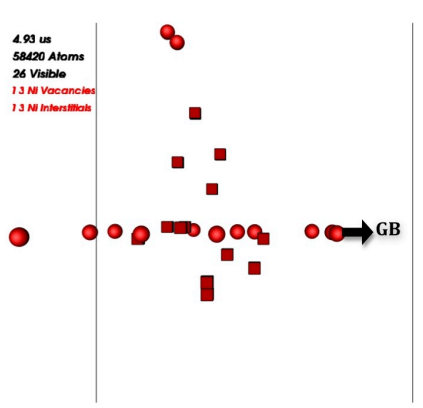

(b)
Figure 2: Evolution of radiation damage in the fcc Ni $\Sigma 5$ system, (a) shows the damage after the MD simulation, (b) after the otf-KMC (In all figures sphere is an interstitial and cube is a vacancy).

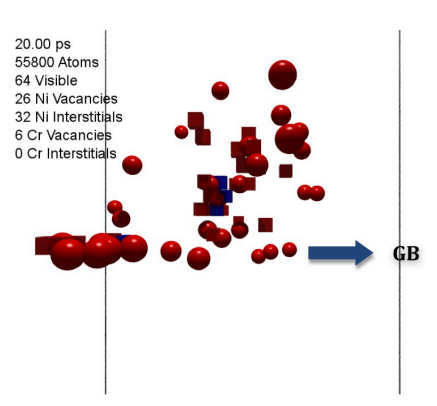

(a)

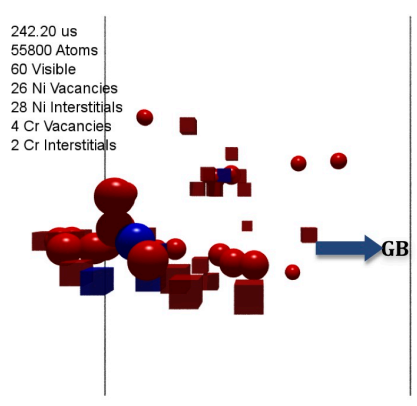

(b)
Figure 3: Evolution of radiation damage in the Ni-Cr $\Sigma 3$ system, (a) shows the damage after the MD simulation, (b) after the otf-KMC (In all figures sphere is an interstitial and cube is a vacancy).

In the $\Sigma 5$ GB alloy system most of the defects accumulated at the GB. During the otf-KMC simulation vacancies were observed to migrate quickly to the GB, if they were near the GB. Most defects further away either annihilated by recombination between vacancies and interstitials or migrated towards the GB. Figure(4) shows snapshots of the system immediately after the collisions cascade and after the otf-KMC.

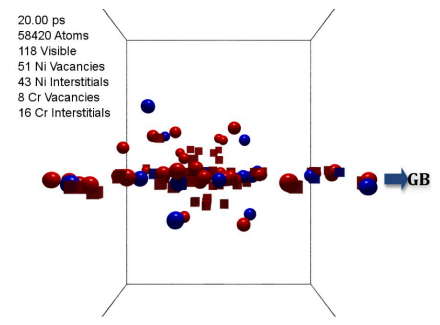

(a)

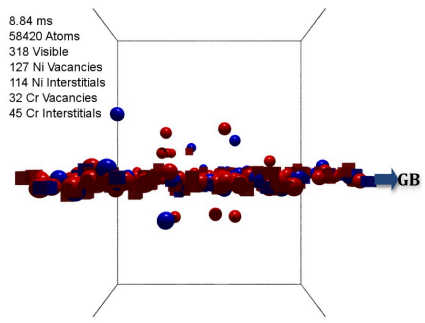

(b)
Figure 4: Evolution of radiation damage in the Ni-Cr $\Sigma 5$ system, (a) shows the damage after the MD simulation, (b) after the otf-KMC (In all figures sphere is an interstitial and cube is a vacancy).

For the case of $55 \mathrm{~GB}$ in the alloy system, we found that the number of defects increased during the otf-KMC simulation. We investigated this, as one would expect that the number

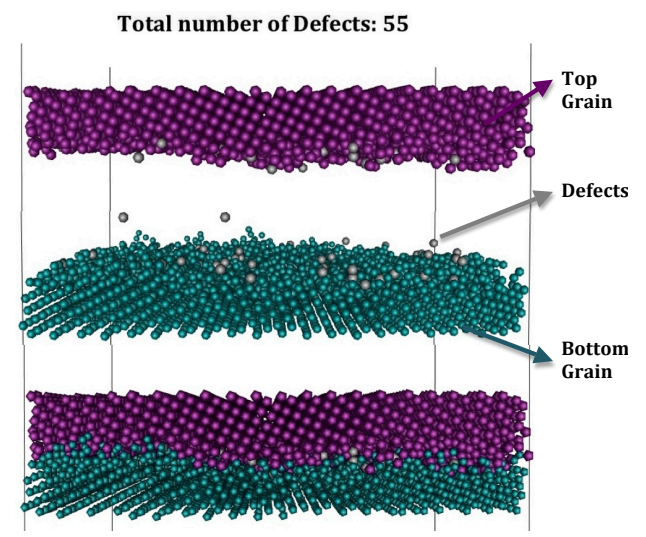

Figure 5: The roughening of the GB for the $\Sigma 5$ alloy system observed after long timescale evolution of the radiation damage.

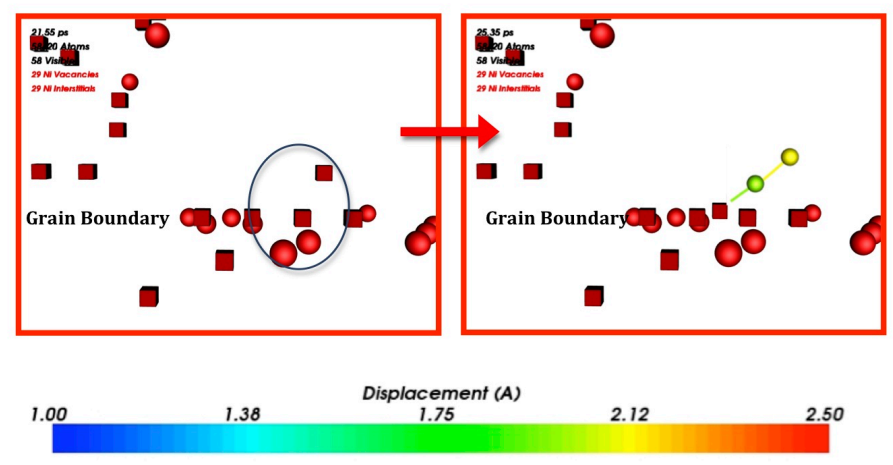

Figure 6: The emission of an interstitial from the GB to combine with vacancy sitting near the GB (In all figures sphere is an interstitial and cube is a vacancy).

of defects would decrease because of recombinations between vacancies and interstitials. We extended the coincident site lattices of the top and bottom grains to investigate whether any roughening happens in the GB during the evolution of the collision cascade. The GB at the start of the simulation is clearly defined by a plane. Due to the displacement and migration of atoms sites during the simulation sites that were part of the top GB become part of the bottom GB, thus the GB can no longer be defined by a plane. We observed that there was a large amount of roughening at the GB for this system and the number of real defects decreased from 306 defects to only 55 defects, as shown in figure (5). Roughening was also observed for the $\Sigma 5 \mathrm{~GB}$ in the pure Ni system but to a smaller extent.

A number of interesting transitions were observed during the otf-KMC simulations, which illustrate the power of these methods for the evolution of radiation damage. In agreement with the work of Bai et al. [15] we observed a number of cases where interstitials were emitted from the GB to recombine with a vacancy sitting near GB. An example of such an event is shown in figure (6).

A number of transitions involving concerted motions of interstitials were observed in the simulations. These include recombinations with vacancies in the bulk, where the vacancy and interstitial are separated by a few angstroms, for example see 
figure (7(a)). The diffusion of an interstitial towards a GB as shown in figure (7(b)) and the diffusion of interstitials in the bulk as shown in figure $(7(\mathrm{c}))$.

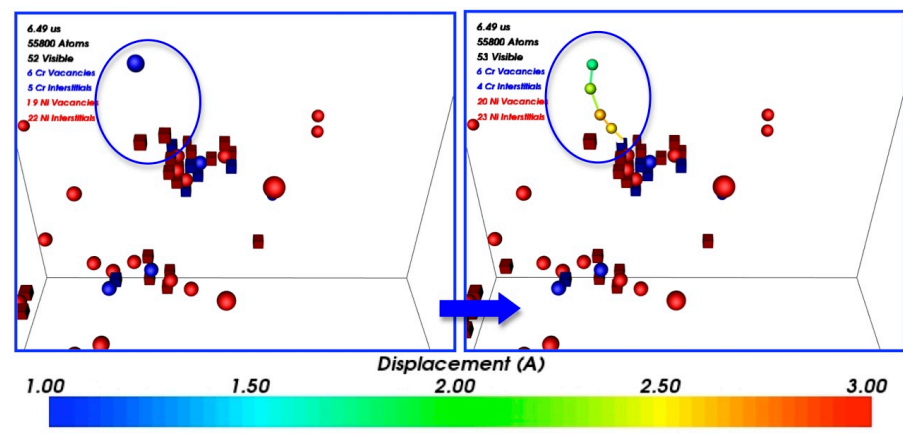

(a)

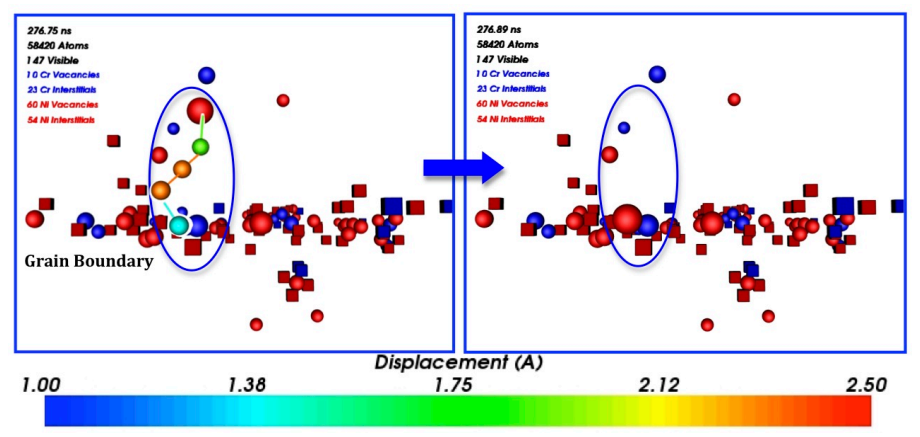

(b)

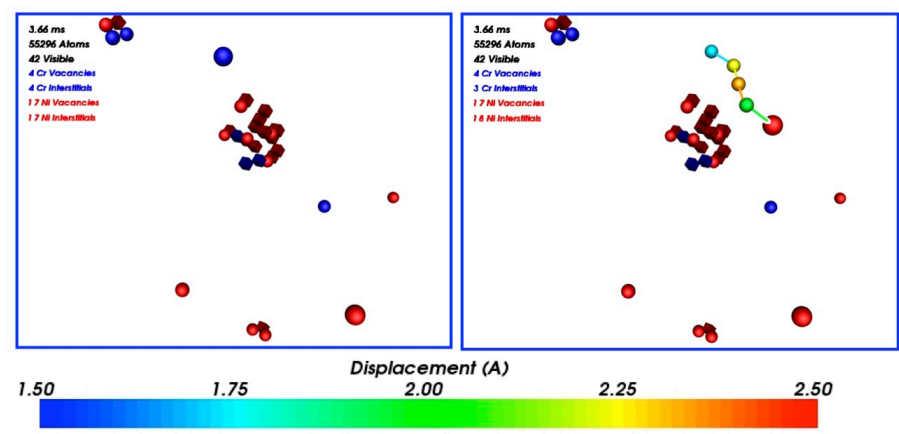

(c)

Figure 7: Examples of concerted movements of interstitials during the otf-KMC phase of the simulation. (a) shows a long range recombination of a vacancy and an interstitial, (b) shows the migration of an interstitial to a GB in a single hop and (c) shows the migration of an interstitial in the bulk (In all figures sphere is an interstitial and cube is a vacancy).

\section{Conclusions}

Otf-KMC simulations of the diffusion of vacancies to GBs show that in most cases a vacancy is found to be attracted to the GB and diffuses towards it with a typical barrier of $0.9 \mathrm{eV}$. This was also observed during the simulation of the evolution of collision cascades near GBs, but it also observed that vacancies that are far enough from GBs have the possibility to form vacancy clusters.

Results for the bulk systems and the $\Sigma 3$ twin boundary were found to be similar in both pure fcc $\mathrm{Ni}$ and the $\mathrm{Ni}-\mathrm{Cr}$ alloy. In these cases vacancy clusters were observed to form, which are relatively immobile. Interstitial diffusion dominates the motion of the defects and there are quite a lot of interstitial-vacancy recombinations that occur during the simulations.

For the $\Sigma 5$ GB most of the defects resulting from the collision cascades were found to accumulate at the GB during the collision cascade itself. For the defects that are not formed at the GB a combination of the recombination of interstitials and vacancies and the diffusion of these defects to the GBs was observed in most cases. Interstitials are observed to be far more mobile than vacancies and always were found to do one of these two things. Vacancies diffuse over a much longer timescale and were also observed to cluster, when formed far enough away from the GB. Substantial GB roughening was observed during the evolution of the radiation damage, particularly in the $\mathrm{Ni}-\mathrm{Cr}$ alloy system.

During the otf-KMC simulations numerous concerted motion transitions occurred, many of which were linked to important transitions in the systems. Examples of these include the emission of an interstitial from the GB to recombine with a vacancy sitting in the bulk, with a low barrier comparable to that of the vacancy migration barrier in the bulk. The ability of the otf-KMC to capture such events was very important and this provides insight into moves that need to be included in atomic $\mathrm{KMC}$ and object KMC approaches.

The presence of large vacancy clusters in the simulations causes the simulations to become less efficient, this is due to the presence of many low barriers. In this work these were addressed by blocking these transitions as they did not contribute to any net translation of the clusters.

\section{Acknowledgement}

Zainab Al Tooq is grateful to the European project Perform 60 and Loughborough University for funding for a PhD studentship. Computing time for the project was provided by Loughborough high performance computing centre. We are also grateful to Roger Smith for useful discussions.

[1] R. Smith, D. E. Harrison Jr., and B. J. Garrison, Phys. Rev. B 40, 93 (1989).

[2] D. Mulliah, S. D. Kenny, and Roger Smith, Modeling of stick-slip phenomena using molecular dynamics, Physical Review B 69, 205407 (2004).

[3] G. Henkelman, and H. Jónsson, Long time scale kinetic Monte Carlo simulations without lattice approximation and predefined event table. The Journal of Chemical Physics 115, 21 (2001), 9657.

[4] L. J. Vernon, and S. D. Kenny, and R. Smith et al. (2011) Growth mechanisms for $\mathrm{TiO}_{2}$ at its rutile (110) surface. In Physical Review B 83 (7).

[5] C. Scott, S. Blackwell, L. Vernon, S. Kenny, Michael Walls, and Roger Smith: Atomistic surface erosion and thin film growth modelled over realistic time scales, J. Chem. Phys. 135, 174706 (2011).

[6] S. Blackwell, R. Smith, S. D. Kenny and J. M. Walls: Modeling the sputter Deposition of Thin Film Photovoltaics using Long Time Scale Dynamics Techniques, Mater. Res. Soc. Symp. Proc. Vol. 1327 (C)2011 Materials Research Society, DOI:10.1557/opl.2011.1124.

[7] T.R. Allen, L. Tan, G.S. Was, E.A. Kenik, Thermal and radiation-induced segregation in model Ni-base alloys, Journal of Nuclear Materials, Volume 361, Issues 2-3, 15 April 2007, Pages 174-183.

[8] Z. Jiao, J.T. Busby, G.S. Was, Deformation microstructure of protonirradiated stainless steels, Journal of Nuclear Materials, Volume 361, Issues 2-3, TMS 2007: Wechsler Symposium, Proceedings of the Symposium on Radiation Effects, Deformation and Phase Transformations in 
Metals and Ceramics, organized in honor of Prof. Monroe S. Wechsler, 15 April 2007, Pages 218-227.

[9] Y. Mishin: Atomistic modeling of the gamma and gamma' phases of the Ni-Al system , Acta Mater., 2004, 52, 1451-1467.

[10] Pär Olsson, Janne Wallenius, Christophe Domain, Kai Nordlund, and Lorenzo Malerba, Erratum: Two-band modeling of $\alpha$-prime phase formation in Fe-Cr [Phys. Rev. B 72, 214119 (2005)]

[11] Giovanni Bonny. FeNiCr potential and its properties (September 2010).

[12] Ziegler JF, Biersack JP, Littmark U, The Stopping and Range of Ions in Matter (Pergomon, New York, 1985).

[13] Swope, W. C. A computer simulation method for the calculation of equilibrium constants for the formation of physical clusters of molecules: Application to small water clusters. The Journal of Chemical Physics 76, 1 (1982), 637.

[14] G. Henkelman, B.P. Uberuaga, and H. Jónsson, A climbing image nudged elastic band method for finding saddle points and minimum energy paths, J. Chem. Phys., 113, 9901 (2000).

[15] Xian-Ming Bai, Arthur F. Voter, Richard G. Hoagland, Michael Nastasi, and Blas P. Uberuaga, Efficient Annealing of Radiation Damage Near Grain Boundaries via Interstitial Emission Science, 327, 1631-1634 (2010). 